\title{
Electrocoagulation and Subsequent Recovery of Phenolic Compounds
}

\author{
Weerachai Phutdhawong, Sombat Chowwanapoonpohn, and Duang Buddhasukh ${ }^{\dagger}$
}

\author{
Department of Chemistry, Faculty of Science, Chiang Mai University 50202, Thailand
}

(Received March 21, 2000; Accepted June 26, 2000)

Electrocoagulation is an electrochemical technique with many applications, in which a variety of unwanted dissolved particles and suspended matter can be effectively removed from an aqueous solution by electrolysis. Thus, for example, it is used to clarify food wastewater, ${ }^{1}$ tar-sand and oil-shale wastewater, ${ }^{2}$ as well as potable water. ${ }^{3}$ It can remove phosphate from sewage, ${ }^{4,5}$ cyanide and chromate from industrial wastewater, ${ }^{6}$ and fluoride from drinking water. ${ }^{7}$ Several heavy metal ions can also be efficiently removed from industrial waste..$^{8,9}$ Electrocoagulation can also be used to decolorize dyecontaining solutions, such as textile wastewater ${ }^{10,11}$ and crude aqueous plant extracts. ${ }^{12-17}$ The main advantages of the electrocoagulation method over coagulation by the addition of chemicals, such as salts of aluminium and iron, are simple equipment and easy operation, a shortened reactive retention period, a reduction or absence of equipment for adding chemicals, and a decreased amount of precipitate or sludge. Moreover, and perhaps most important of all, during electrocoagulation the liquid is not enriched with anions, and its salt content does not increase, as is the case of a chemical treatment. $^{6}$ In this study, we decided to investigate the electrocoagulation of certain phenolic compounds in a systematic manner, in view of the fact that phenolic substances often occur in many industrial wastes, especially textile and paper mill effluents, and also in many plant extracts, e.g. flavonoids and tannins. Additionally, we also report on a simple method for recovering these phenolic substances from coagulated sludge.

\section{Experimental}

Two aluminium plates (dimension $30 \times 10 \times 0.05 \mathrm{~cm}$ ) were used as electrodes. These were dipped $3 \mathrm{~cm}$ apart and $9 \mathrm{~cm}$ deep into a magnetically-stirred aqueous solution (1 litre) of a phenolic compound $(0.1 \% \mathrm{w} / \mathrm{v})$ in a glass jar (diameter $11 \mathrm{~cm}$, height $23 \mathrm{~cm})$. Sodium chloride $(2 \mathrm{~g})$ was added as an electrolyte. Direct current $(0.5 \mathrm{~A}, 22 \mathrm{~V})$ from a DC power supplier was then passed through the solution via the two electrodes. At every 15 -min interval during a 2 -h period of electrolysis, a $10-\mathrm{cm}^{3}$ aliquot sample of the solution was drawn, filtered, and taken for an absorbance measurement at an appropriate wavelength of the absorption maximum for each phenolic compound, as follows: phenol 250, resorcinol 272, pyrocatechol 252, pyrogallol 243, phloroglucinol 233, $n$-propyl 3,4,5-trihydroxybenzoate 243 , orcinol 241 , hydroquinone 243 , and tannin $242 \mathrm{~nm}$. The measured absorbance was then converted to the residual weight percentage of the compound by a calibration curve obtained from a plot between the absorbance

$\doteqdot$ To whom correspondence should be addressed. versus the concentration for each compound. All of the phenolic compounds used were of standard reagent grade, and were used as such. Phenol and resorcinol (1,3dihydroxybenzene) were purchased from Fluka Chemie AG, Buchs, Switzerland; pyrocatechol (1,2-dihydroxybenzene) and pyrogallol (1,2,3-trihydroxybenzene) were purchased from E. Merck, Darmstadt, Germany; phloroglucinol (1,3,5trihydroxybenzene) and $n$-propyl 3,4,5-trihydroxybenzoate were purchased from BDH, Dorset, UK; orcinol (1-methyl-3,5dihydroxybenzene) was purchased from Aldrich Chemical Co., St. Louis, MO, USA; and hydroquinone (1,4-dihydroxybenzene) and tannin from May \& Baker Ltd., Manchester, UK.

\section{Results and Discussion}

It was previously proposed that the main reactions occurring during the electrolysis of this type typically produce aluminium ion at the sacrificial anode and hydroxide ion as well as hydrogen at the cathode, as shown below: 18,19

Anode reaction: $\mathrm{Al} \rightarrow \mathrm{Al}^{3+}+3 \mathrm{e}^{-}$,

Cathode reaction: $2 \mathrm{H}_{2} \mathrm{O}+2 \mathrm{e}^{-} \rightarrow 2 \mathrm{OH}^{-}+\mathrm{H}_{2}$.

The resulting in situ formation of gelatinous aluminium hydroxide, $\mathrm{Al}(\mathrm{OH})_{3}$, thus effects the coagulation or coprecipitation of particulates from the solution by adsorption. The aluminium ion liberated from the anode may also interact directly with an anion or a phenol, which then precipitates out of the solution in the form of an insoluble salt, e.g. aluminium triphenolate $\left[\mathrm{Al}(\mathrm{OAr})_{3}\right]$.

However, with the current results obtained as shown in Fig. 1, in which plots of the residual weight percentage versus the electrolysis time for aqueous solutions of the phenolic compounds under study are compared, it is quite evident that there can be a degree of selectivity in complexation of the phenols with the aluminium ion, given a suitable condition, a phenomenon hitherto unreported. It can thus be seen that the phenolic compounds studied neatly fall into two categories: one stays more or less unaffected in the solution $(\leq 8 \%$ precipitation for phenol, orcinol, and phloroglucinol, and $\leq 25 \%$ precipitation for resorcinol and hydroquinone); another is almost completely precipitated from the solution (i.e. more than $94 \%$ precipitation for pyrocatechol, pyrogallol, $n$-propyl 3,4,5-trihydroxybenzoate, and tannin). It can thus be seen that the former category consists of the phenol, itself, and phenols with 1,3-dihydroxy, 1,3,5-trihydroxy, and 1,4-dihydroxy substitution patterns, while the latter category comprises phenols (including tannin) with 1,2-dihydroxy and 1,2,3-trihydroxy substitution patterns.

This suggests that the interaction of the type shown below 


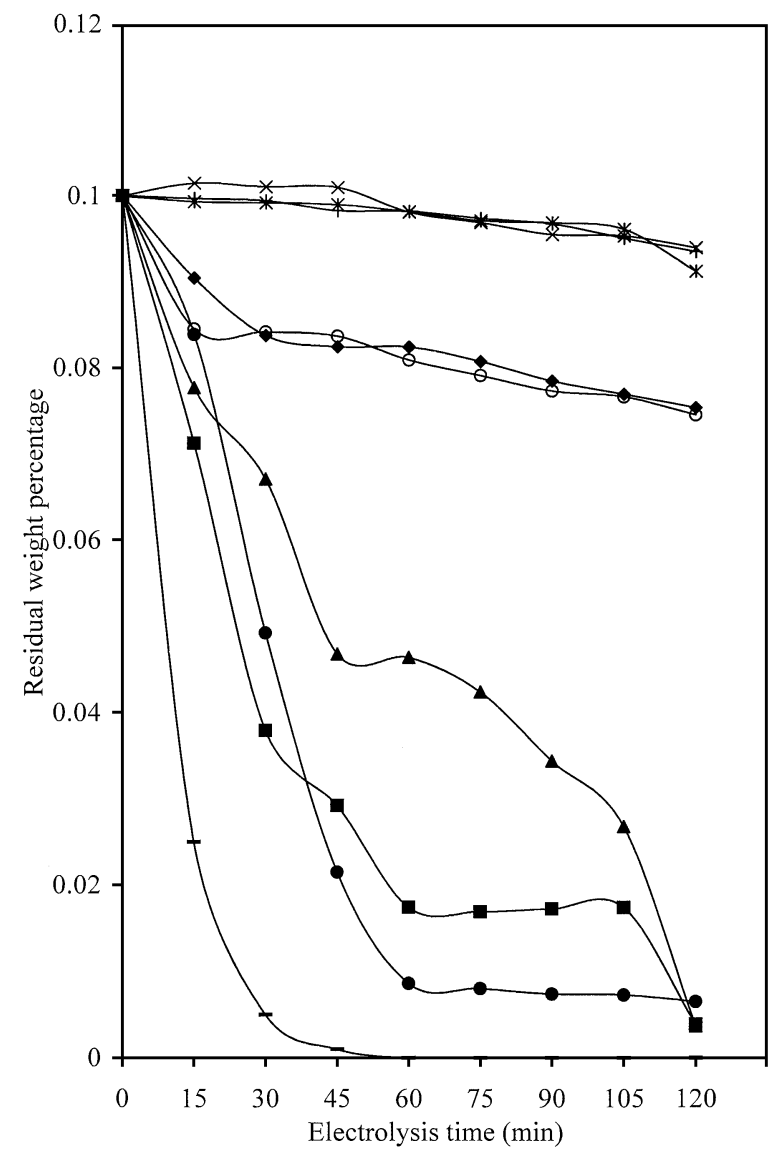

Fig. 1 Plot of the residual weight percentage and electrolysis time for each phenolic compound: $*$, phenol; $\bullet$, resorcinol; $\mathbf{\Lambda}$, pyrocatechol; $\mathbf{\square}$, pyrogallol; $\times$, phloroglucinol; $\bullet, n-$ propyl 3,4,5-trihydroxybenzoate; +, orcinol; $\bigcirc$, hydroquinone; - , tannin.

seems to be important for preferential precipitation, even at neutral $\mathrm{pH}$. This is, of course, possible only with those phenols with at least two adjacent (1,2-disubstituted) hydroxyl groups.<smiles></smiles>

Previous experiments and operations involving electroprecipitation or electrocoagulation have focused entirely on eliminating by the adsorption and coprecipitation of certain dissolved or suspended materials without trying to recover them. However, in some circumstances, those materials may constitute the very part that is desired. In this study, we used a simple method to recover them. This involves dissolving the precipitate, which may consist of adsorbed substances on aluminium hydroxide gel, or precipitated substances in the form of insoluble aluminium salts, or both, in diluted (5-10\%) hydrochloric acid solution. The acid effectively liberates the adsorbed substances, presumably by converting the adsorbing aluminium hydroxide into non-absorbing aluminium chloride, or by breaking down the insoluble aluminium salts of the phenolic substances into aluminium chloride and free phenolics. The obtained acidic solution, which should now consist of aluminium chloride and the liberated substances, is then subjected to extraction with a small quantity of 1-butanol. Evaporation of the alcohol then affords the adsorbed or precipitated compounds directly, leaving aluminium chloride in the aqueous solution. By doing this with pyrocatechol, pyrogallol, and $n$-propyl 3,4,5-trihydroxybenzoate, the original compounds were recovered $(60-92 \%)$ from the precipitate obtained by electrolysis. This was confirmed by identical melting points, TLC and IR of the compounds before and after electrolysis (with tannin, however, a slight discrepancy in the two m.p.'s was obtained, probably due to the non-homogeneous nature of the tannin). This suggests that the phenolic compounds are not preferentially oxidized at the electrode under the above-mentioned electrolytic conditions.

\section{Conclusion}

Electrocoagulation has proved to be quite useful and practical in many operations that constitute the elimination of certain unwanted particles or chemical species from an aqueous solution. In this study, we showed that the method can also be applied to some phenolic compounds. In addition, there is a degree of selectivity in this kind of precipitation among these compounds. Moreover, the precipitated compounds can be recovered quite satisfactorily by a simple method.

\section{Acknowledgements}

TRF (Thailand Research Fund) is gratefully acknowledged for providing the RGJ (Royal Golden Jubilee) Grants for W. P. and S. C.

\section{References}

1. E. C. Beck, A. P. Giannini, and E. R. Ramirez, Food Technol., Feb. 1974, 18.

2. R. R. Renk, Energy Prog., 1988, 8, 205.

3. E. A. Vik, D. A. Carlson, A. S. Eikum, and E. T. Gjessing, Water Res., 1984, 18, 1355.

4. E. Dobolyi, Water Res., 1978, 12, 1113.

5. O. Groterud and L. Smoczynski, Water Res., 1986, 20, 667.

6. V. E. Cenkin and A. N. Belevtsev, Effluent Water Treat J., 1985, 25, 243.

7. N. Mameri, A. R. Yeddou, H. Lounici, D. Belhocine, H. Grib, and B. Bariou, Water Res., 1998, 32, 1604.

8. J. Mrozowski and J. Zielinski, Environ. Protection Eng., 1983, $9,77$.

9. D. R. Jenke and F. E. Diebold, Water Res., 1984, 18, 855.

10. T. R. Demanin and K. D. Uhrich, Am. Dyestuff Rep., 1988, 77, 13.

11. J. S. Do and M. L. Chen, J. Appl. Electrochem., 1994, 24, 785.

12. K. Miwa, Jpn. Kokai Tokkyo Koho 78,105,500; Chem. Abstr., 1979, 90, 3392y.

13. K. Miwa, S. Maeda, and Y. Murata, Jpn. Kokai Tokkyo Koho 79 89,066; Chem. Abstr., 1979, 91, 173681e.

14. K. Miwa, S. Maeda, and Y. Murata, Jpn. Kokai Tokkyo Koho 79 90,199; Chem. Abstr., 1979, 91, 206904k.

15. J. Adduci, D. Buddhasukh, and B. Ternai, J. Sci. Soc. Thailand, 1987, 13, 179.

16. W. Phutdhawong and D. Buddhasukh, J. Sci. Fac. CMU, 1998, 25, 87.

17. W. Phutdhawong and D. Buddhasukh, ACGC Chem. Res. Commun., 2000, 10, 61.

18. L. Ming, Y. Sunrui, H. Zhangiun, Y. Bina, L. Wel, P. Liu, and F. Kefichero, Fluoride, 1983, 20, 54.

19. J. Adduci, Honours Thesis, 1984, La Trobe University, Australia. 